\title{
Avances en el desarrollo de un clasificador de imágenes termográficas de planta del pie diabético basado en una red neuronal de retropropagación
}

José Federico Ramírez Cruz, Edmundo Bonilla Huerta, Lauro Reyes Cocoletzi, José Crispín Hernández Hernández

Instituto Tecnológico de Apizaco, Apizaco, Tlaxcala,

México

\begin{abstract}
Resumen. Actualmente el incremento de enfermedades crónicas (diabetes) y el diagnostico preventivo en el campo de la medicina nos llevan a usar la tecnología para prevenir complicaciones a largo plazo o incluso a predecir con un rango de confiabilidad el porcentaje de probabilidad a ser propensos a estas patologías. En la literatura médica se tiene una relación entre la temperatura de algunas zonas de las plantas del pie en relación a el flujo sanguíneo y el grado de azúcar en este, por lo que en este trabajo se presenta una propuesta para la implementación de una red neuronal (tipo retropropagación) que será capaz de realizar la clasificación de patrones de imágenes digitalizadas en base a la variación térmica e identificar patrones para la clasificación de angiosomas planares de individuos sanos y posibles individuos diabéticos no diagnosticados (a través de anormalidades en la lectura de la imagen térmica).La red neuronal de retropropagación implementada tiene la característica que pre-clasifica a través del almacenamiento de los pesos de los patrones de entrenamiento, para evaluar la mayor relación (a través de la correlación de Pearson) existente entre las distintas imágenes evaluadas lo que conlleva a un mayor grado de confiabilidad en la clasificación.
\end{abstract}

Palabras clave: Correlación de Pearson, red neuronal de retropropagación, angiosomas planares.

\section{Advances in the Development of a Thermographic Image Classifier of Diabetic Foot Plant Based on Backpropagation Neural Network}

\begin{abstract}
Currently the increase in chronic sickness (diabetes) and preventive diagnosis in the medical field lead us to use technology to prevent long-term complications or even to predict with a range of reliability the percentage of probability to be prone to these pathologies. In the medical literature there is a relationship between the temperature of some areas of the plant in relation to the blood flow and the degree of sugar in the foot, so in this paper we present a proposal for the implementation of a neural network (Type backpropagation) that will be able to perform the classification of patterns of digitized images based on
\end{abstract}




\begin{abstract}
the thermal variation and to identify patterns for the classification of plantar angiosomes of healthy individuals and possible undiagnosed diabetic individuals (through abnormalities in the reading of the Thermal imaging). The implemented backpropagation neural network has the characteristic that pre-classifies through the storage of the weights of the training patterns, to evaluate the greater relation (through the Pearson correlation) existing between the different evaluated images which leads to a higher degree of reliability in the classification.
\end{abstract}

Keywords: Pearson correlation, backpropagation neural network, planar angiosomes.

\title{
1. Introducción
}

El pie tiene importantes funciones como la sustentación del peso corporal, la postura bípeda, el equilibrio y la locomoción, condiciones indispensables para la mayoría de las actividades del ser humano. La vitalidad de sus estructuras depende de su sistema arterial, cuyo elemento central es el arco plantar profundo, del que se originan las arterias metatarsianas plantares, fundamentales para la irrigación de los dedos y de la región plantar profunda. En el paciente sano el flujo arterial al pie es redundante, pero no es así en el paciente isquémico [R. Fernández-Samos Gutiérrez, 2012].

La teoría del angiosoma explica las variaciones que existen en los aportes de sangre arterial a la piel y estructuras adyacentes entre las diferentes regiones del cuerpo, proporciona la base para interpretar muchos procesos fisiológicos y patológicos [R. Fernández-Samos Gutiérrez, 2012].
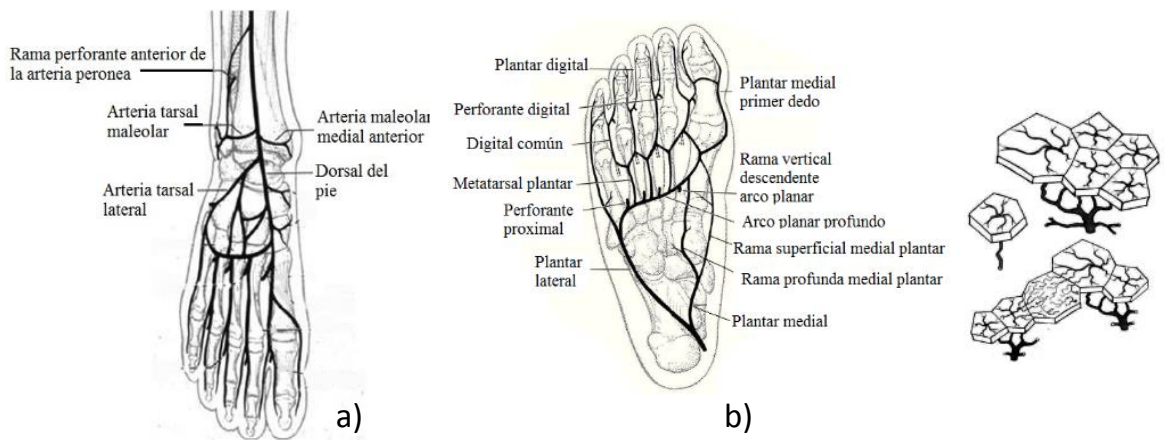

a)

b)

Fig. 1. a) Arterias presentes en el pie, b) arterias planta del pie con sus colaterales, c) esquema de angiosoma.

El objetivo de este trabajo es presentar avances en la cuantificación de anormalidades presentes en la imagen térmica de las plantas de los pies a través de una termografía (la termografía arroja características de la irrigación de la sangre que a su vez influye en la temperatura alrededor de la arteria por la teoría del angiosoma) que nos permita evaluar y cuantificar defectos congénitos o patologías para la prevención sin tener que realizar un análisis invasivo sobre el paciente [Neville et al. 2009]. 


\section{Estado del arte}

Algunos puntos importantes en la toma y análisis de las imágenes (muestreo) se retoman en base a trabajos previos existentes, estos se consideran a continuación:

Las imágenes térmicas se representan en colores RGB que a su vez se ligan a una escala en grados centígrados $\left({ }^{\circ} \mathrm{C}\right)$. A través de imágenes térmicas de ambos pies, se pueden identificar diferencias entre áreas correspondientes del pie derecho y el pie izquierdo con el fin de aportar información cuantitativa acerca de la distribución de la temperatura plantar, por ejemplo la información se obtiene mediante la segmentación de cada imagen térmica en cuatro regiones del angiosoma planar y a través de una clasificación de los píxeles se obtienen regiones diferentes que cubre un área planar $[\mathrm{H}$. Peregrina et al. 2013].

Investigaciones con termografía relacionan la existencia de una asociación positiva entre una alta diferencia asimetría térmica de los angiosomas planares contra el riesgo de padecer diabetes tipo 2 o ulceraciones que conlleven a problemas de amputación [Neves et al. 2015].

En pies diabéticos la ocurrencia de una ulcera es frecuentemente asociada con la hipertermia. La hipertermia se define como una temperatura superior a $2.2^{\circ} \mathrm{C}$ en una región dada de uno de los pies comparada con la temperatura de la misma región del pie contralateral observable en la termografía [L. Vilcahuaman et al. 2014].

Cabe mencionar que en el estudio realizado por $\mathrm{K}$. Ammer encontró que las deformidades presentes en los dedos (callosidades o varices) no influyen directamente o en mayor medida en la variación de la temperatura censada en los pies con la termografía o con los riesgos en la ulceración de los pies, aunque se debe tomar en consideración estas deformidades para considerar en otras patologías [K. Ammer et al. 2001].

Para pacientes diabéticos diagnosticados el mayor riesgo corresponde la ulceración de los pies, posteriormente infecciones vasculares y culminar con amputación de miembros. La termografía es un medio de bajo costo para determinar complicaciones vasculares debidas al riesgo de ulceraciones en la planta del pie. Esto se lleva en base a la relación ente la osteomielitis correlacionada por la imagen infrarroja y el estudio clínico que valide los resultados obtenidos con termografía [J.R.Harding et al. 1998].

\section{Metodología}

Se toman imágenes térmicas (formato jpg) a través de una cámara Fluke serie $13 \mathrm{ABC}$ a una muestra aleatoria doble (dos fotogramas térmicos por cada sujeto) de 20 individuos en un rango de edad de 20 - 23 años estas imágenes son procesadas digitalmente y son la base para el entrenamiento y pruebas de la programación del clasificador a través de una red neuronal de retropropagación por medio de mecanismos de computo.

Para la adquisición de las imágenes térmicas se propone dar suficientemente tiempo al sujeto de prueba el equilibrar la temperatura ambiente con la temperatura de sus pies (un mínimo de 15 minutos) en un espacio donde la temperatura sea de $23^{\circ} \mathrm{C} \pm 0.3$ durante la toma de la imagen. Colocar al individuo en posición horizontal y aislar con un fondo negro los pies para prevenir radiación externa que afecte la captura de la 
imagen. La cámara infrarroja se puede colocar a $1.1 \mathrm{~m}$ de distancia de los pies del sujeto pero en general depende de la resolución (en pixeles) de la cámara con la que se cuente [L. Vilcahuaman et al. 2014].

La red de retropropagación en una red neuronal supervisada tiene limitantes inherentes como lo es el tiempo de aprendizaje basado en el número de iteraciones (épocas) para su entrenamiento en las salidas deseadas así como variaciones en la salida, pero es bastante buena para clasificar pues su lentitud es compensada con el grado de fiabilidad en el momento de clasificar, esto principalmente, debido a la supervisión de pequeñas cambios de los pesos hasta llegar al entrenamiento y aprendizaje deseado.

Inicialmente se plantea digitalizar la imagen e implementar como entrada a la red neuronal cada pixel y su información de forma individual, esto es, si la imagen termografía tiene una resolución de $750 \times 450$ pixeles el número de pixeles a procesar es de 337,500 pixeles en tres capas RGB (337,500 de profundidad 3) pero debido a la alta demanda de computo necesario se descarta esta opción y se utilizan regiones térmicas identificables (alrededor de unos cientos de pixeles a procesar como se presenta más adelante) en las muestra obtenidas de las imágenes digitalizadas.

Estas regiones son las zonas frías, calientes y templadas en el área del pie, obtenidas por la cámara termográfica.

La obtención de las regiones frías, calientes y templadas se realiza primero separando el fondo de la forma (la forma es la imagen termográfica del pie), esto se lleva a cabo realizando una transformación de los planos de imagen RGB al formato estándar de imagen YIQ, así se obtienen los pixeles que aportan la luminancia ( $\mathrm{Y}=$ luminancia $)$ que pertenecen a la forma y nunca al fondo (esto debido a que la toma de muestra se realiza en un ambiente controlado).

En base a la posición de los pixeles obtenidos de la forma se generan seis cuadrantes como se muestra en la figura 2, para delimitar el uso de pixeles a las regiones que contienen la información de interés.

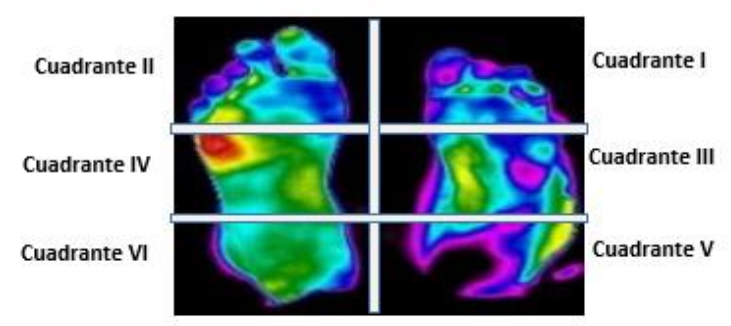

Fig. 2. Ilustración de los cuadrantes definidos para analizar las regiones de interés.

Conociendo la posición de los pixeles que pertenecen a la forma se procede a obtener los valores de la posición de estos pixeles en los tres planos por separado cada uno (valor de la posición del pixel en el plano $\mathrm{R}$, en el plano $\mathrm{G}$, en el plano B). Esta información se almacena en 3 matrices y se evalúa el valor mayor de la posición de los 3 planos y se asigna como el valor predominante y así obtener las áreas templadas, frías y calientes de la imagen termográfica. 
Las entradas hacia la red neuronal constan básicamente de la información obtenida de las áreas frías, templadas y calientes en los seis cuadrantes en los que se divide la imagen, así, cada cuadrante tendrá puntos de interés representados por la cantidad de áreas con tonos rojos, verdes y azules. Esto representa la distribución de la temperatura en la imagen y esta información es la base para evaluar el grado de similaridad (para este análisis se usan las dos regiones calientes, templadas y frías de mayor área en cada cuadrante, si se desea se pueden utilizar mayor número de áreas en cada cuadrante).

Se aplica un patrón de entrada como estímulo para la primera capa de las neuronas de la red, se va propagando a través de todas las capas superiores hasta generar una salida, se compara el resultado en las neuronas de salida con la salida que se desea obtener y se calcula un valor de error para cada neurona de salida. A continuación, estos errores se transmiten hacia atrás, partiendo de la capa de salida hacia todas las neuronas de la capa intermedia que contribuyan directamente a la salida [Freeman et al. 1991].

Este proceso se repite, capa por capa, hasta que todas las neuronas de la red hayan obtenido un error que describa su aportación relativa al error total. Basándose en el valor del error dado, se reajustan los pesos de conexión de cada neurona, de manera que se almacena estos pesos para el patrón aprendido y en la siguiente vez que se presente el mismo patrón o similar se reconocerá inmediatamente, para el caso de que sea más de un patrón se almacenaran los pesos aprendidos para el siguiente patrón, entonces con respecto a la salida que esté más cercana a la deseada de los pesos de los patrones obtenidos se clasificara de forma más rápida.

Se propone el almacenar los pesos de entrenamiento obtenido para cada uno de los patrones a clasificar para obtener una categorización optimizada en la relación de un patrón nuevo de entrada.

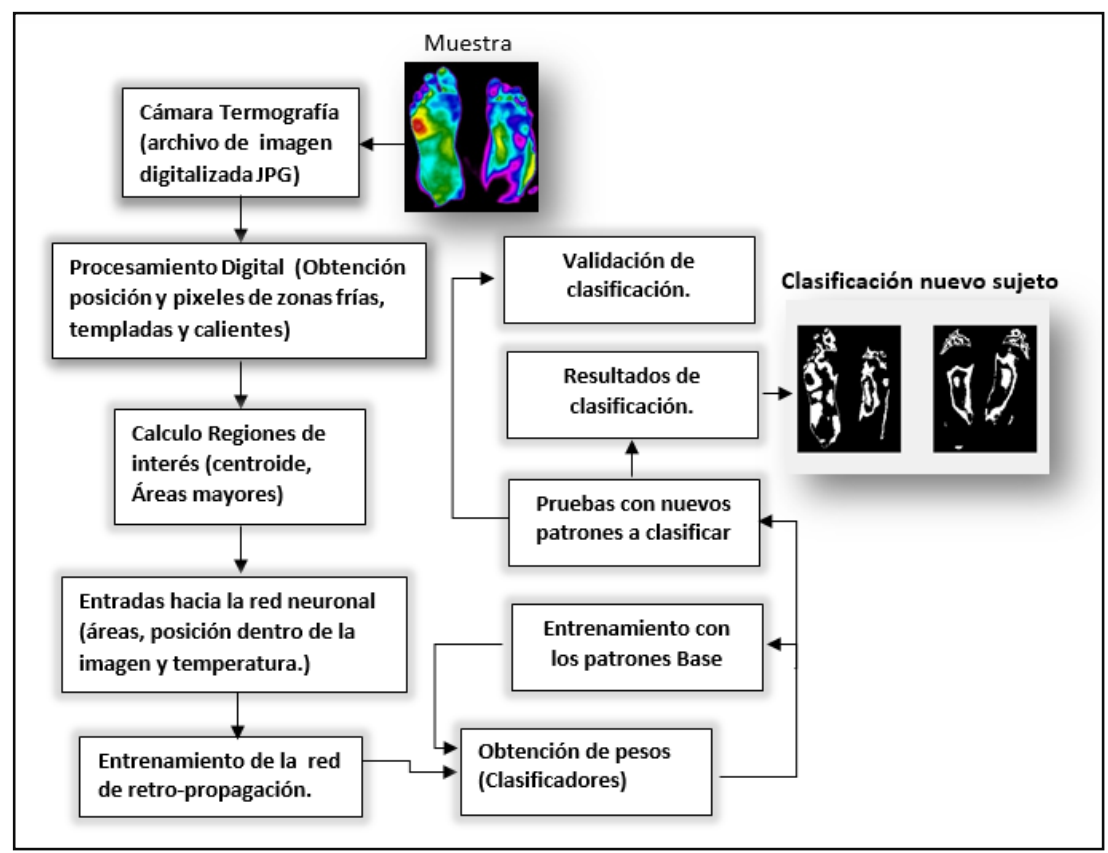

Fig. 3. Diagrama a bloques de operación del algoritmo. 


\subsection{Clasificación y distribución de las regiones de interés en la termografía}

Imagines digitalizadas. Las imágenes digitalizadas tienen 5 patrones base deseadas con la que se entrenó a la red neuronal (salida deseada donde el error tienda a cero), las demás imágenes que sean introducidas a la red neuronal deben clasificarse de acuerdo a la similaridad que tengan con respecto a estos patrones base.

Estos patrones se toman como base porque representan características muy distintas unas de otras del conjunto de muestreo obtenido de los sujetos de prueba y sirven para representar las anormalidades que se podrán encontrar en pacientes por diagnosticar o ya diagnosticados.

Con los 5 patrones base se entrena a la red neuronal para la clasificación de 5 características definidas en la distribución térmica de la planta de los pies.

Estas características se definen como un rango normal y cuatro irregularidades presentes en las muestras de los sujetos para clasificar, la descripción de las categorías se presentan a continuación.

Patrón 1. - Al tomar una muestra con la cámara termográfica lo ideal es que se presente simetría en ambas plantas de los pies, como en ocasiones esto no es posible el clasificador también considera este caso, es decir, si algún prueba se clasifica en este patrón quiere decir que se debe realizar otra toma de muestra del paciente pues la termografía carece de la simetría mínima requerida para clasificar.

Patrón 2.-Simetria mínima en umbral, analiza las regiones de los falanges y la zona angular de la planta del pie, establece patrón sano.

Patrón 3.-Pie plano, característica de deformación congénita tratable y candidato a estudios clínicos para establecer propensión a la diabetes. Para el patrón 3, el pie plano influye en encontrar las características de los angiosomas y la probabilidad de presentar un cuadro clínico de diabetes por lo que se recomendaría realizar un estudio de la sangre.

Patrón 4.-Anormalidad 1 en la distribución de la temperatura debido a problemas en los angiosomas, pie de sujeto con posible padecimiento diabético.

Patrón 5.-Anormalidad 2 en la distribución de la temperatura de los angiosomas debido a problemas de circulación de sangre, posible cuadro diabético.

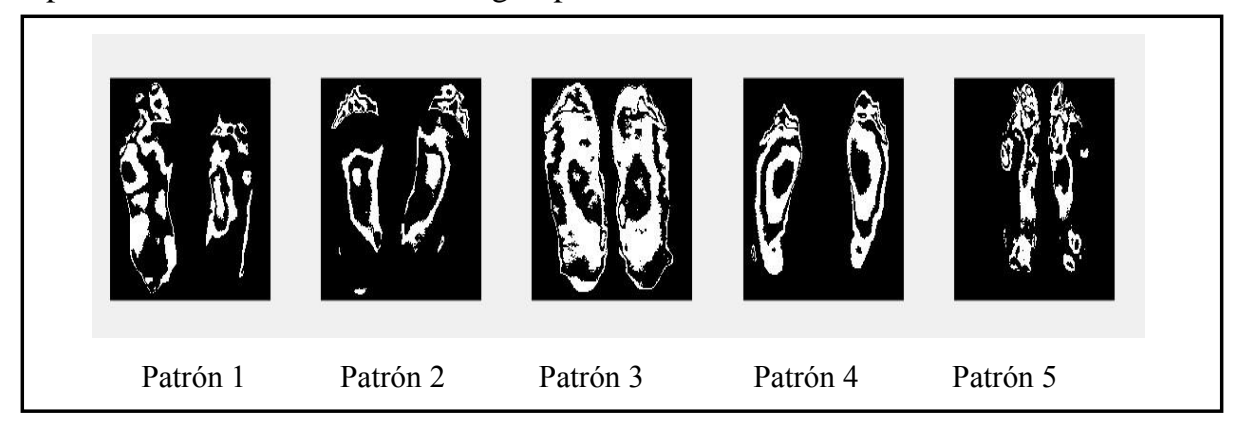

Fig. 4. Patrones base para la clasificación de la salida de la red neuronal. 
La binarización de las imágenes se emplea para resaltar en la salida y desplegar resultados obtenidos de la clasificación y solo es de carácter de interface visual al usuario.

La obtención de las áreas o regiones de interés (total de pixeles) que conforman las regiones térmicas distribuidas en la planta del pie se obtienen una vez realizado el procesamiento digital de la imagen, estas áreas y su posición en la imagen definen las entradas de la red neuronal.

Se establecen los valores de entrada con respecto a el área de los pixeles encontrados en los seis cuadrantes utilizados por ejemplo, Área $1=1500$ pixeles, Área $2=2058$ pixeles, Área $3=3156$ pixeles, Área $4=1204$ pixeles, Área $5=3560$ pixeles y Área Total = sumatoria de todas las áreas anteriores. Se divide a cada una de las regiones por un factor de 100000 o cercano al número de pixeles totales que forman a la imagen para obtener una representación proporcional de los pixeles que conforman a las distintas regiones en valores positivos $<1$. Esto se realiza por que la red neuronal utiliza datos continuos para el cálculo de los pesos.

Entonces, con esto tendremos una representación de los pixeles que conforman a la imagen y no a cada pixel de manera individual, al final un conjunto de pixeles en una imagen presentan características similares y esto es lo que se obtiene al detectar y procesar las regiones de interés.

Para este caso de estudio se trabaja con 12 regiones para cada imagen y la sumatoria total de estas regiones. Al final la suma de las regiones indica el total de pixeles que se han detectado y que son relevantes de la imagen.

La salida obtenida se clasifica de acuerdo a la correlación de cada salida obtenida con respecto a las salidas aprendidas y clasificadas utilizando el coeficiente de correlación de Pearson, por lo que la red neuronal proporciona la información y clasifica pero el coeficiente de correlación de Pearson interpreta esa información y valida el resultado.

\section{Pruebas y resultados obtenidos}

Se introducen las imágenes de prueba para observar el funcionamiento del clasificador.

Tabla 1. Parámetros de salida de la red neuronal durante el entrenamiento de los patrones base para la clasificación.

\begin{tabular}{|c|c|c|}
\hline $\begin{array}{l}\text { Patrones de } \\
\text { entrenamiento }\end{array}$ & $\begin{array}{l}\text { Salida red neuronal } \\
\text { Patrones binarizada } \\
\text { (deseada) }\end{array}$ & Salida entrenamiento (obtenido) \\
\hline$\# 1$ & {$\left[\begin{array}{lllll}1 & 0 & 0 & 0 & 1\end{array}\right]$} & -0.00269 \\
\hline$\# 2$ & {$\left[\begin{array}{lllll}0 & 0 & 1 & 0 & 0\end{array}\right]$} & {$\left[\begin{array}{lllll}-0.000105 & 0.0191 & 0.9985 & 0.0452 & -0.0001\end{array}\right]$} \\
\hline \#3 & {$\left[\begin{array}{lllll}1 & 0 & 1 & 1 & 0\end{array}\right]$} & {$\left[\begin{array}{lllll}0.9991 & 0.00048 & 1 & 0.99893 & 0.00020\end{array}\right]$} \\
\hline \#4 & {$\left[\begin{array}{lllll}0 & 1 & 0 & 0 & 1\end{array}\right]$} & {$\left[\begin{array}{lllll}-0.9290 & 0.99897 & -0.8998 & -0.3172 & 0.99904\end{array}\right]$} \\
\hline$\# 5$ & 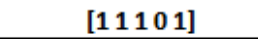 & [ [ $\left.\begin{array}{lllll}0.99896 & 0.99999 & 0.99904 & 0.03998 & 0.99999\end{array}\right]$ \\
\hline
\end{tabular}

La red neuronal procesa la información dada y clasifica de acuerdo a la mayor similitud que encuentre en los puntos calientes, templados y fríos distribuidos en la 
imagen. Se clasifica como uno de los cinco patrones con base en los pesos obtenidos en el entrenamiento de la red neuronal.

La tabla 1, muestra los valores de trabajo e información puntual de los patrones de entrenamiento de la red neuronal como son la discretización en parámetros binarios de las salidas deseadas.

La tabla 2 a su vez muestra resultados arrojados para 5 patrones de prueba que clasifico la red neuronal, el error y el factor de correlación que definió a que patrón corresponde para su clasificación.

Tabla 2. Resultados de 5 patrones de prueba y el error.

\begin{tabular}{|c|c|c|c|}
\hline $\begin{array}{l}\text { Patrones } \\
\text { de prueba }\end{array}$ & Salida entrenamiento & $\begin{array}{c}\text { Correlacion de } \\
\text { Pearson }\end{array}$ & Error (comparacion salida deseada- salida obtenida) \\
\hline \#6 & 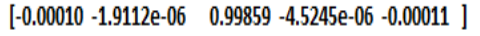 & 0.867 & 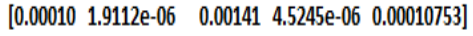 \\
\hline$\# 7$ & $\left.\begin{array}{llll}1 & 0.99936 & -0.00130 & 0.99999\end{array}\right]$ & 0.8856 & {$\left[\begin{array}{lllll}0.00068 & 0 & 0.00064 & 0.0013029 & 1 \mathrm{e}-05\end{array}\right]$} \\
\hline \#8 & {$\left[\begin{array}{lllll}-0.00169 & 0.98841 & 0.00048 & 0.00028 & 0.98895\end{array}\right]$} & 0.875 & {$\left[\begin{array}{lllll}0.00170 & 0.01159 & 0.00048 & 0.00028 & 0.01105\end{array}\right]$} \\
\hline \#9 & 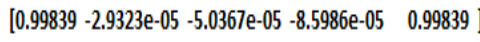 & 0.908 & 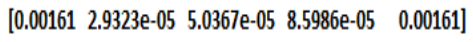 \\
\hline$\# 10$ & {$\left[\begin{array}{lllll}0.94341 & -0.00077 & 0.99998 & 0.95196 & -0.13268\end{array}\right]$} & 0.9423 & 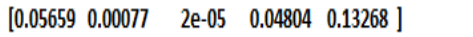 \\
\hline
\end{tabular}

La respuesta de salida de clasificación obtenida con el clasificador y lo observado de forma visual cualitativa arroja resultados con variación como se muestra en la figura 5 , aunque debe de considerarse la región de interés que está tomando el clasificador corresponde a la distribución térmica de las zonas calientes, templadas y frías.

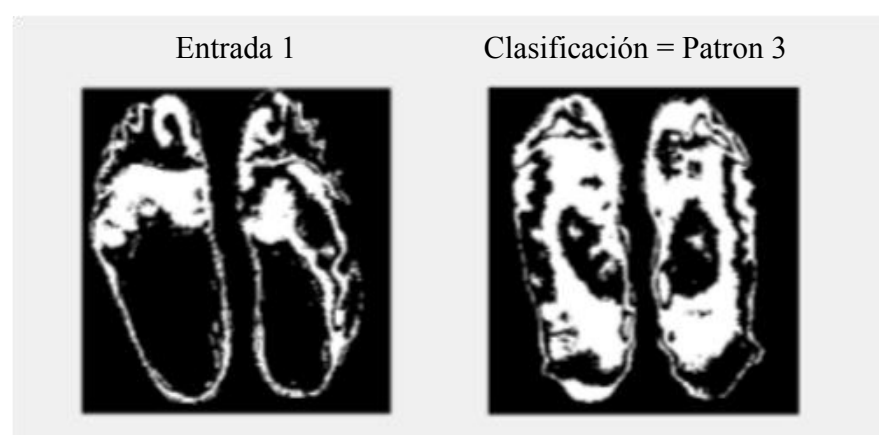

Fig. 5. Resultados arrojados por el clasificador, se clasifican como pertenecientes al patrón 3.

El resumen de los resultados de clasificación de las muestras obtenidas de los sujetos de prueba se muestra en la tabla 3.

Cabe resaltar que se introducen por cada sujeto dos muestras y el resultado esperado es que replique la clasificación del patrón de la muestra 1 con la muestra 2 , sin embargo en algunos casos no sucede esto. Esto se debe a variaciones entre una muestra y otra en algunos casos sutiles y en otros casos no tanto de ahí que se realiza la validación de los resultados obtenidos a través la validación cruzada de n-iteraciones, obteniendo resultados de una tasa de error de $0.88 \%$, lo que significa que tiene aún muchas áreas de oportunidad para la mejora del clasificador, pues idealmente se necesita obtener una 
Avances en el desarrollo de un clasificador de imágenes termográficas de planta del pie diabético ...

tasa de error menor al $0.05 \%$ para denotar que el clasificador discretiza con un porcentaje muy alto de validación.

Tabla 3. Resultados de clasificación de los sujetos de prueba, en la muestra 1 y la muestra 2.

\begin{tabular}{c|l|l|l|l|l|l|l|l|l|l|l|l|l|l|l|l|l|l|l|l}
\hline Sujeto de prueba & 1 & 2 & 3 & 4 & 5 & 6 & 7 & 8 & 9 & 10 & 11 & 12 & 13 & 14 & 15 & 16 & 17 & 18 & 19 & 20 \\
\hline $\begin{array}{c}\text { Muestra 1 (clasificacion arrojada } \\
\text { por la red neuronal) }\end{array}$ & 2 & 3 & 2 & 1 & 1 & 1 & 1 & 2 & 3 & 2 & 4 & 5 & 5 & 4 & 3 & 2 & 1 & 1 & 2 & 5 \\
\hline $\begin{array}{c}\text { Muestra 2 (clasificacion arrojada } \\
\text { por la red neuronal) }\end{array}$ & 2 & 2 & 1 & 4 & 3 & 1 & 1 & 1 & 3 & 3 & 3 & 5 & 1 & 4 & 2 & 2 & 1 & 1 & 3 & 5 \\
\hline \\
\hline \begin{tabular}{c} 
Desviacion estandar muestra 1 \\
\hline Desviacion estandar muestra 2
\end{tabular} \\
\hline
\end{tabular}

Se agrega solo como referencia la desviación estándar de los resultados de la distribución de las muestras en cuanto a cuál sería la dispersión de la clasificación de los sujetos de prueba, esto está limitado pues el rango de cambio no es muy grande ya que solo se puede clasificar dentro de cinco patrones posibles.

\section{Conclusiones}

La estabilización de una red neuronal de retropropagación necesita de ciertas características adicionales e inherentes a ella para clasificar de forma adecuada, que pueden ser lógica difusa, Bayes Ingenuo, Control clásico o estadística.

Lo importante es fusionar las redes neuronales con otras metodologías para obtener un resultado optimizado.

Para nuestro caso el coeficiente de correlación de Pearson utiliza las salidas y los 5 patrones de entrenamiento para llevar a cabo la clasificación de las salidas, es decir, se soporta la red neuronal con un análisis estadístico.

Además, se implementa el almacenamiento de pesos con base a los patrones a clasificar por lo que se puede ser una ventaja en algunos casos el entrenar exclusivamente para un patrón y en algunos otros casos una desventaja pero esto dependerá de la aplicación a realizar.

\section{Trabajo a futuro}

Continuar con el desarrollo del clasificador tomando en cuenta los tres planos de colores RGB y la relación con la variación térmica $\left({ }^{\circ} \mathrm{C}\right)$, tratar digitalmente la información a través de técnicas como ecualización de histograma, filtrado digital entre otras técnicas para definir las áreas de interés para obtener mejores resultados a través de un pre-procesamiento de la imagen más robusto.

Un avance mayor seria que agrupara patrones nuevos incrementando automáticamente las opciones de clasificación, es decir, no solo se contara con cinco 
patrones posibles a clasificar si no que automáticamente aumentara el número de patrones según se encontraran nuevas características en la distribución de las zonas térmicas del pie.

Realizar cálculos estadísticos de acuerdo a la distribución de las áreas de interés y que afectan en mayor medida al diagnóstico del paciente y no solo de forma cualitativa.

Obtener imágenes termografías de pacientes diagnosticados con diabetes positivo y evaluar el clasificador con estos sujetos de prueba.

Hacer más robusto el clasificador para la detección de ulceraciones y no solo tomar en consideración el procesamiento de las variaciones de temperatura en las plantas de los pies.

Evaluar la posibilidad de tomar toda la información de la imagen termográfica (todos los pixeles) con técnicas robustas como el cómputo paralelo o el uso de GPU’s.

\section{Referencias}

1. Peregrina, H., Morales, L. A.: Thermal image processing for quantitative determination of temperature variations in plantar angiosomes. In: IEEE International Instrumentation and Measurement Technology Conference (2013)

2. Neves, E. B., Almeida, J., Rosa, C., Vilaça, J., Reis, V. M., Mendes, R.: Anthropometric Profile and Diabetic Foot Risk: A Cross-Sectional Study Using Thermography. In: 37th Annual International Conference of the IEEE Engineering in Medicine and Biology Society (2015)

3. Vilcahuaman, L., Harba, R., Canals, R., Zequera, M., Wilches, C., Arista, M. T., Torres, L., Arbañil, H.: Detection of Diabetic Foot Hyperthermia by Infrared Imaging. In: Engineering in Medicine and Biology Society 36th Annual International Conference of the IEEE (2014)

4. Ammer, K., Melnizky, P., Rathkolb, O., Ring, E. F.: Thermal Imaging Of Skin Changes On The Feet Of Type II Diabetics. Engineering in Medicine and Biology Society In: Proceedings of the 23rd Annual International Conference of the IEEE (2001)

5. Harding, J. R., Wertheim, D. F., Williams, R. J., Melhuish, J. M., Banerjee, D., Harding, K. G.: Infrared Imaging in Diabetic Foot Ulceration. Engineering in Medicine and Biology Society. In: Proceedings of the 20th Annual International Conference of the IEEE (1998)

6. Neville, R., Attinger, E. C.: Revascularizacion de angiosomas específicos en isquemia critica de la extremidad: ¿Es importante la arteria diana? Anales de cirugía Vascular, revista internacional de Cirugía vascular y Terapéutica Endovascular (2009)

7. Fernández, R.: El modelo angiosoma en la estrategia de revascularización de la isquemia crítica. Servicio de Angiología y Cirugía Vascular, Complejo Asistencial Universitario de León, España (2012)

8. Freeman, J. A., Skapura, M. D.: Neural Networks: Algorithms, Applications, and Programming Techniques. California, U.S., Addison-Wesley Publishing Company (1991)

9. Mitchell, T. M., Ryszard, S., Michalski, J. G.: Machine Learning. New York, US, McGraw Hill (1996) 